\title{
TECNOLOGIAS DIGITAIS PARA CAPACITAÇÃO DE PROFISSIONAIS DE ENFERMAGEM SOBRE SEGURANÇA DO PACIENTE: REVISÃO INTEGRATIVA
}

\author{
DIGITAL TECHNOLOGIES FOR TRAINING NURSING PROFESSIONALS ON PATIENT \\ SAFETY: INTEGRATIVE REVIEW
}

\author{
Valeska Tais de Araújo Hoffmann ${ }^{1} *$ Desirée Zago Sanchis $^{2} *$ Patricia Aroni $^{3} *$ Dêmely \\ Biason $^{4}$ Ferreira Valéria Rodrigues Godoi ${ }^{5} *$ Maria do Carmo Fernandez Lourenço Haddad $^{6}$
}

\begin{abstract}
RESUMO
Objetivo: analisar as evidências científicas sobre o uso de tecnologias digitais para capacitação de profissionais de enfermagem sobre segurança do paciente. Método: revisão integrativa, com busca de estudos primários indexados nas bases de dados Literatura Latino-Americana e do Caribe em Ciências da Saúde, SciVerse Scopus, Cumulative Index to Nursisng and Allied Health Literature, Web of Science e Medical Literature Analysis and Tetrievel System Online, publicados em inglês, português e espanhol, sem limite de tempo. Resultados: foram selecionados 11 artigos, distribuídos entre os anos de 2008 a 2020. As tecnologias digitais desenvolvidas foram softwares, módulos de educação online, vídeos e slides, com os seguintes temas: comunicação, análise de fármacos, instrumentação cirúrgica, erros e interrupções de medicações, administração de medicamentos e infecções. e Conclusão: as tecnologias digitais utilizadas para a capacitação dos profissionais de enfermagem mostraram-se eficazes para transmissão e consolidação do conhecimento com a finalidade de proporcionar melhores práticas em saúde.
\end{abstract}

Palavras-chave: Segurança do paciente; Enfermagem; Tecnologia; Educação Continuada; Aprendizagem

\begin{abstract}
Objective: The article aims to analyze the scientific evidence on the use of digital technologies for training nursing professionals on patient safety. Method: integrative review, with search for primary studies indexed in the Latin American and Caribbean Literature in Health Sciences, SciVerse Scopus, Cumulative Index to Nursisng and Allied Health Literature, Web of Science and Medical Literature Analysis and Tetrievel System Online databases, published in English, Portuguese and Spanish, with no time limit. Results: 11 articles were selected, distributed between the years 2008 to 2020. The digital technologies developed were software, online education modules, videos and slides, with the following themes: communication, drug analysis, surgical instrumentation, errors and interruptions of medications, medication administration and infections. Conclusion: the digital technologies used to train nursing professionals proved to be effective in transmitting and consolidating knowledge in order to provide better health practices.
\end{abstract}

Keyword: Patient Safety; Nursing; Technology; Education Continuing; Learning

\footnotetext{
${ }^{1}$ Universidade Estadual de Londrina, Londrina, Paraná/Brasil, https://orcid.org/0000-0003-4102-364X

${ }^{2}$ Universidade Estadual de Londrina, Londrina, Paraná/Brasil, https://orcid.org/0000-0001-8066-1113.

${ }^{3}$ Universidade Estadual de Londrina, Londrina, Paraná/Brasil, https://orcid.org/0000-0001-5092-2714.

${ }^{4}$ Universidade Estadual de Londrina, Londrina, Paraná/Brasil, https://orcid.org/0000-0003-1591-1213.

${ }^{5}$ Universidade Estadual de Londrina, Londrina, Paraná/Brasil, https://orcid.org/0000-0001-7668-4427.

${ }^{6}$ Universidade Estadual de Londrina, Londrina, Paraná/Brasil, https://orcid.org/0000-0001-7564-8563.
} 


\section{INTRODUÇÃO}

A segurança do paciente é caracterizada como um conjunto de ações que tem por finalidade prevenir e evitar danos associados aos cuidados em saúde. ${ }^{1}$ Durante a assistência em serviços de saúde, potenciais riscos devem ser reduzidos a um grau admissível prezando pela segurança do paciente. $^{2}$ Desde 1999, o assunto ganhou evidência por meio da publicação do relatório nominado To Err is Human, o qual divulgou dados coletados pelo Institute of Medicine dos Estados Unidos, onde se evidenciou que os erros relacionados ao cuidado ocasionavam de 44 a 98 mil eventos adversos anualmente. ${ }^{3}$

Após a publicação do relatório, houve fortalecimento das discussões sobre a educação na área da saúde com o objetivo de minimizar os eventos adversos, e por conseguinte como resultado, a efetivação de publicações de políticas e estratégias de segurança do paciente. No Brasil destaca-se a oficialização da Portaria GM/MS $\mathrm{n}^{\circ}$ 1996/2007, que dispõe sobre as diretrizes para a implementação da Política Nacional de Educação Permanente em Saúde (PNEPS), que tem por finalidade orientar os profissionais acerca das práticas do cuidado aos usuários. Também foram adotadas pelas organizações de saúde ministrar capacitações para os colaboradores de maneira participativa e reflexiva ${ }^{4}$, modelo de ensino que visa o desenvolvimento técnico-prático do profissional, como também de competências e habilidades, fortalecendo práticas seguras e redução de eventos adversos. $^{5}$

Assim como a PNEPS, a Educação em Saúde (ES) se mostra como importante ferramenta para promoção da segurança do paciente, pois visa a conscientização individual e coletiva dos profissionias. ${ }^{6}$ A ES é uma ação desenvolvida por profissionais da saúde, dentre eles o enfermeiro, com a finalidade de conscientizar sobre a saúde, bem como desenvolver a percepção como participante ativo na manutenção de seus cuidados. $^{7}$

Em conjunto com as estratégias e políticas educacionais, modernas tecnologias estão sendo utilizadas em ambientes destinados ao desenvolvimento de profissionais de enfermagem, com intuito de disseminar informações de maneira direcionada e instantânea. ${ }^{8}$ As Tecnologias de Informação e Comunicação (TIC) têm possibilitado difundir conteúdos com fins pedagógicos, além de viabilizar a autoaprendizagem via internet, fortalecendo as melhores práticas assistenciais. ${ }^{8}$

Assim como a TIC, os Objetos Virtuais de Aprendizado (OVA) também são aplicados como suporte de ensino direcionados, que permitem a apreensão da temática proposta e aprendizagem mais interativa. Os OVA são definidos como 
unidades de aprendizagem constituído por sons, imagens, animações e documentos, que podem ser associados a internet. ${ }^{9}$

Portanto, o uso de tecnologias digitais permite um processo de ensinoaprendizagem de profissionais de enfermagem mais dinâmico, individualizado ou coletivo, de maneira participativa, que acompanha o ritmo de cada indivíduo, com potencial para subsidiar uma formação de maior qualidade, viabilizando práticas assistenciais mais seguras e humanizadas. ${ }^{10}$

O estudo tem por objetivo analisar as evidências científicas sobre o uso de tecnologias digitais para capacitação de profissionais de enfermagem sobre segurança do paciente.

\section{MÉTODOS}

Trata-se de uma revisão integrativa da literatura, metodologia de pesquisa que compila relevantes publicações de maneira sistematizada e ordenada, proporciona $\mathrm{O}$ aprimoramento da temática, possibilita formular conclusões e evidenciar lacunas de conhecimento. $^{11}$

A investigação ocorreu conforme as etapas definidas para esta metodologia, iniciando pela elaboração da questão norteadora; estratégia de busca e extração de dados; categorização; análise dos estudos incluídos; síntese dos resultados e apresentação da revisão. ${ }^{12}$
Para elaboração do protocolo, optouse por utilizar a estratégia de $\mathrm{PICo}^{13}$, (acrônimo para População, Intervenção e Contexto) onde $\mathrm{P}=$ Profissionais de enfermagem, $\mathrm{I}=$ Tecnologia e $\mathrm{Co}=$ Segurança do Paciente, apresentando a seguinte questão norteadora: Quais as evidências disponíveis na literatura científica sobre o uso de tecnologias digitais para capacitação de profissionais de enfermagem sobre segurança do paciente?

A coleta de dados foi realizada durante os meses de julho e agosto de 2020, nas seguintes bases de dados indexadas: Literatura Latino-Americana e do Caribe em Ciências da Saúde (LILACS), SciVerse Scopus (SCOPUS), Cumulative Index to Nursisng and Allied Health Literature (CINAHL), Web of Science (WOS), e Medical Literature Analysis and Tetrievel System Online (Medline via PubMed), por meio do portal de periódicos da Coordenação de Aperfeiçoamento de Pessoal de Nível Superior (CAPES). Os descritores utilizados nas buscas foram: "Profissionais de enfermagem", "Tecnologia" e "Segurança do Paciente" no Banco de Descritores em Ciências da Saúde (DeCS) para a base de dados em língua portuguesa, e "Nurses", "Technology" e "Patient safety" extraídos no Medical Subject Headings (MeSH) para as bases de dados em língua inglesa. Foi utilizado o operador booleano "AND" para as 
buscas em português e inglês, que favoreceu o cruzamento dos termos para a busca.

Os critérios de inclusão estabelecidos foram: artigos originais completos em português, inglês e/ou espanhol, sem filtro para ano de publicação, e que abordavam o assunto. Estudos secundários como revisões, relato de experiência, reflexão, teses, dissertações, capítulo de livros, artigos teóricos, carta ao editor, resumos de trabalhos publicados em anais de eventos e artigos que não respondiam a questão norteadora foram excluídos deste estudo.

Foi utilizado o Software State of the Art Throungh Systematic Review (START),

Figura 1 - Fluxograma de seleção dos artigos primários incluídos na revisão integrativa. Londrina, PR, Brasil, 2020

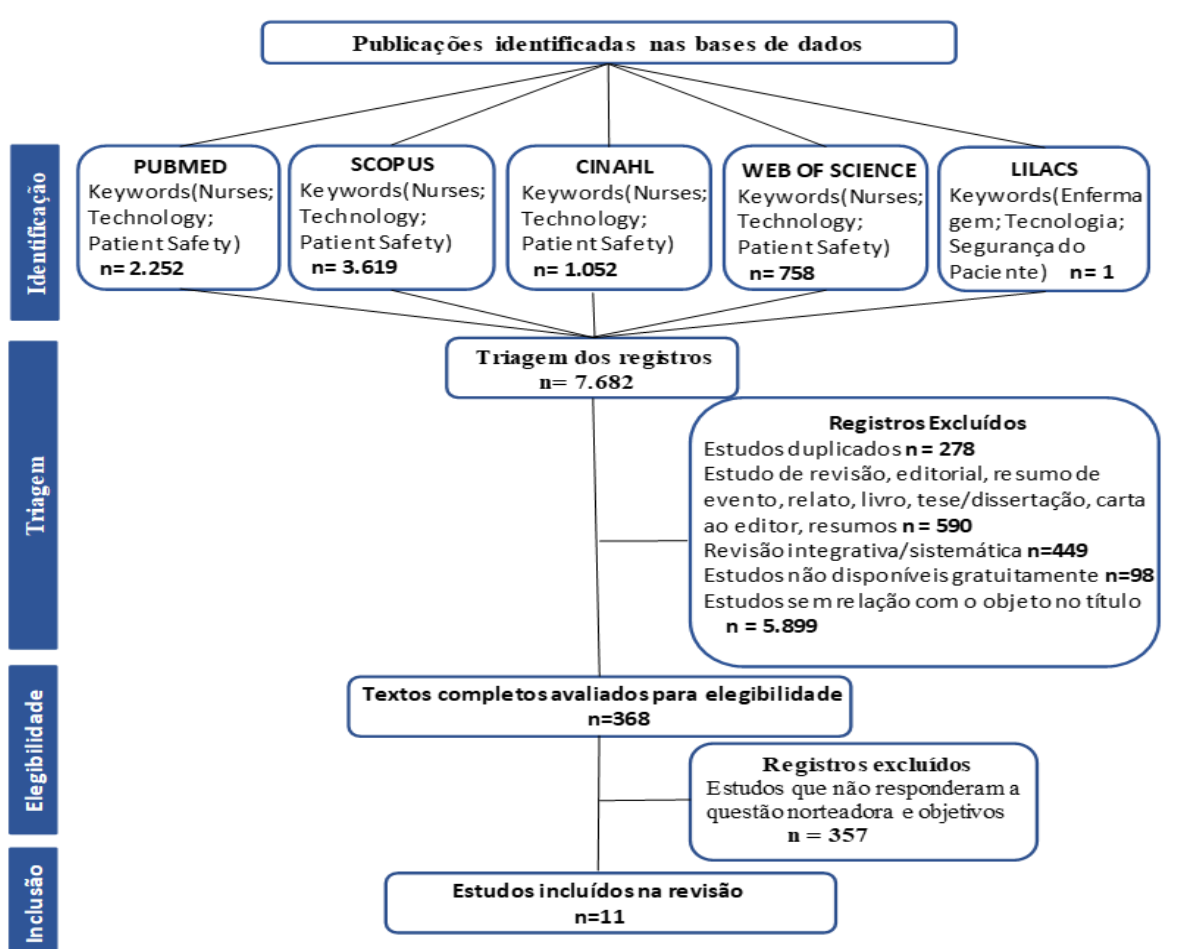

Fonte: Os autores como apoio ao planejamento, execução e análise dos resultados. ${ }^{14}$

A leitura dos artigos durante as etapas deste estudo foi realizada em pares, os quais não apresentaram discordância na seleção dos artigos para inclusão na revisão. $\mathrm{Na}$ primeira etapa foi realizada a leitura de título e resumo para seleção dos artigos conforme critérios de inclusão e exclusão, posteriormente, leitura na íntegra dos artigos selecionados conforme apresentado na Figura 1. 
Após seleção, os artigos foram divididos em 4 categorias conforme tecnologia utilizada durante a intervenção realizada com profissionais de enfermagem.

Os estudos incluídos nesta revisão foram avaliados quanto aos seguintes níveis de evidência: nível 1 (mais forte) as evidências provenientes de revisão sistemática ou metanálise de ensaios clínicos randomizados; nível 2, evidências derivadas de ensaios clínicos randomizados bem delineados; nível 3, evidências obtidas de ensaios clínicos bem delineados sem randomização; nível 4, evidências provenientes de estudos de coorte e de casocontrole bem delineados; nível 5, evidências originárias de revisão sistemática de estudos descritivos e qualitativos; nível 6, evidências derivadas de um único estudo descritivo ou qualitativo; e nível 7 (mais fraco), evidências oriundas de opinião de especialistas. ${ }^{15}$

\section{RESULTADOS}

Todos os 11 artigos foram escritos na língua inglesa. Em relação as publicações nas bases de dados, um $^{16}$ foi identificado na Web of Science, dois ${ }^{17-18}$ na Medline/Pubmed, $\mathrm{um}^{19}$ na Cinahl, e sete ${ }^{19-26}$ na Scopus. Desses, um ${ }^{17}$ foi publicado em revistas interdisciplinares de saúde, um $^{16}$ em revistas de outras áreas da saúde (medicina, terapeutas e assistentes sociais) e nove ${ }^{18-26}$ em periódicos de enfermagem.
No que tange ao desenho do estudo, $u^{24}$ com abordagem qualitativa, dois ${ }^{17,20}$ estudos randomizado controlado, seis ${ }^{16,18-19,21-}$ 23 estudos randomizado sem controle, e dois ${ }^{25-}$ 26 caso-controle. O quadro 2 apresenta a caracterização dos artigos analisados para a presente revisão.

Em relação aos países de origem dos artigos, observa-se que a maioria foram publicados nos Estados Unidos da América ${ }^{18-}$ 19,21-23,26, seguido da Escócia ${ }^{17,20}$, Alemanha ${ }^{16}$, Finlândia ${ }^{25}$ e Austrália $^{24}$. Ressalta-se que não foram encontrados artigos publicados no Brasil sobre o tema, evidenciando lacunas na literatura nacional.

Referente ao ano de publicação, os estudos estão difundidos entre 2008 e 2020, com maior número de artigos em $2013^{20-21}$ e $2016^{16,23}$, seguido do ano de $2008^{26}, 2014^{18}$, $2015^{25}, 2017^{22}, 2018^{24}, 2019^{17}$ e $2020^{19}$, com apenas um artigo em cada ano citado. Quanto ao nível de evidência, oito ${ }^{15-22}$ estudos foram classificados como nível II, dois ${ }^{25-26}$ como nível IV e um ${ }^{24}$ como nível V.

Os recursos utilizados nas intervenções implementadas nos estudos se enquadram como tecnologia dura, ou seja, todos fizeram uso de equipamentos tecnológicos para capacitação dos profissionais. Três ${ }^{19,24,26}$ estudos aplicaram mais de uma tecnologia, logo, oito ${ }^{16-18,20-23,25}$ estudos adotaram apenas um recurso tecnológico. A seguir, apresentam-se as especificidades das tecnologias e das 
intervenções realizadas nos estudos, classificadas por categorias.

A primeira categoria apresenta $\mathrm{o}$ software, o qual consistiu no manuseio da tecnologia em dispositivos touch screen pelos profissionais. ${ }^{16,19,26}$ Entre os estudos, dois ${ }^{16,26}$ analisaram seus efeitos individualmente, e $\mathrm{um}^{19}$ de maneira mista (individual e em conjunto), por meio de intervenções-padrão com aplicação de questionários antes e após a ação, no entanto, somente uma publicação ${ }^{26}$ descreveu o intervalo de tempo para aplicação do questionário após a capacitação. Em relação ao processo de construção do software, destaca-se a publicação de $2020^{19}$, que descreveu de maneira concisa as etapas percorridas e os profissionais envolvidos.

Os temas abordados nas capacitações foram: cirurgia segura (instrumentação, identificação e contagem) e administração de medicamentos. Os resultados das intervenções apresentaram efeitos positivos, proporcionando melhores práticas e desfechos. Porém, os autores frisaram que as intervenções evidenciadas exigiram orientações prévias para os profissionais sobre o funcionamento de softwares e uso de equipamentos.

A segunda categoria agrupou somente um estudo ${ }^{20}$ que utilizou slides (arquivo PPT), o qual foi aplicada em grupos com aproximadamente 60 minutos em cada sessão, e avaliada por meio de questionário aplicado antes e após seis semanas da intervenção. Os autores descreveram as fases de validação do conteúdo e questionário, como também o estudo piloto. O tema abordado aos profissionais se refere aos princípios básicos na administração de medicamentos de alto risco. A ação revelou respostas satisfatórias, como também maior envolvimento motivacional dos profissionais capacitados referente a manutenção do conhecimento.

$\mathrm{Na}$ terceira categoria foram agrupadas a estratégia de vídeos ${ }^{22-24}$, com os seguintes temas: trabalho em equipe ${ }^{23}$, comunicação $^{23}$, quedas ${ }^{22}$ e interrupção de medicação. ${ }^{24}$ Os vídeos foram reproduzidos em dispositivos tecnológicos ${ }^{22-23}$, ou em plataformas de educação online ${ }^{24}$ para transmissão do conteúdo. Entretanto, o processo de construção do vídeo não foi descrito pelos autores, evidenciando lacunas na informação. A avaliação pós intervenção se sucedeu por meio de grupo focal ${ }^{24} \mathrm{e}$ observadores. $^{22-23}$ Como resultado, este recurso de aprendizagem foi apontado como didático, prático e demonstrou adesão positiva dos profissionais, exibindo diminuição de eventos adversos e incidentes.

A quarta categoria agrupou os $\operatorname{artigos}^{17-19,21,24-26}$ que utilizaram o módulo de educação online. Os participantes foram convidados via e-mail ou folheto para capacitação, e a avaliação pós implementação ocorreram em diferentes períodos. Os módulos poderiam ser acessados 
individualmente a qualquer tempo, no entanto, apresentava prazo determinado para término das sessões. A tecnologia foi descrita pelos profissionais capacitados como acessível, autoexplicativa e pedagógica, fornecendo resultados positivos.

Quadro 1 - Caracterização dos artigos selecionados para a revisão integrativa. Londrina, PR, Brasil, 2020

\begin{tabular}{|c|c|c|c|c|}
\hline Título & $\begin{array}{l}\text { Autor/Ano/País/Base } \\
\text { de dados/Nível de } \\
\text { evidência (NE) }\end{array}$ & $\begin{array}{l}\text { Tecnologias } \\
\text { utilizadas }\end{array}$ & Tema & $\begin{array}{l}\text { Principais } \\
\text { resultados }\end{array}$ \\
\hline $\begin{array}{l}\text { Design and } \\
\text { evaluation of } \\
\text { an interactive } \\
\text { training system } \\
\text { for scrub } \\
\text { nurses } 16\end{array}$ & $\begin{array}{l}\text { Bernhard Glaser; } \\
\text { Tobias Schellenberg; } \\
\text { Lucas Koch; } \\
\text { Mathias Hofer; } \\
\text { Susanne Modemann; } \\
\text { Patrick Dubach; } \\
\text { Thomas Neumuth } \\
2016 \\
\text { Alemanha } \\
\text { Web of Science } \\
N E: 2\end{array}$ & Software & $\begin{array}{l}\text { Instrumentaçã } \\
\text { o cirúrgica. }\end{array}$ & $\begin{array}{l}\text { Passagem correta de } \\
\text { instrumentos; } \\
\text { organização da mesa } \\
\text { cirúrgica. }\end{array}$ \\
\hline $\begin{array}{l}\text { Nurses } \\
\text { knowledge of } \\
\text { high-alert } \\
\text { medications: A } \\
\text { randomized } \\
\text { controlled }_{\text {trial }^{20}}\end{array}$ & $\begin{array}{l}\text { Min-Chin Lu; } \\
\text { Shu Yu; } \\
\text { I-Ju Chen; } \\
\text { Kai-Wei K. Wang; } \\
\text { Hsiang-Fend Wu; } \\
\text { Fu-In Tang } \\
2013 \\
\text { Escócia } \\
\text { Scopus } \\
\text { NE } 2\end{array}$ & $\begin{array}{l}\text { Arquivo PPT } \\
\text { (Slides) }\end{array}$ & $\begin{array}{l}\text { Conceitos } \\
\text { sobre } \\
\text { medicamentos } \\
\text { de alto risco. }\end{array}$ & $\begin{array}{l}\text { Administração e } \\
\text { identificação de } \\
\text { medicamentos de } \\
\text { alto risco; } \\
\text { Motivação da equipe } \\
\text { em adquirir } \\
\text { conhecimento. }\end{array}$ \\
\hline $\begin{array}{l}\text { Clinical } \\
\text { Effectiveness } \\
\text { and Cost of a } \\
\text { Hospital-Based } \\
\text { Fall } \\
\text { Prevention } \\
\text { Intervention }^{22}\end{array}$ & $\begin{array}{l}\text { Teryl K. Nuckols; } \\
\text { Jack Needleman; } \\
\text { Tristan R. Grogan; } \\
\text { Li-Jung Liang; } \\
\text { Pamela Worobel-Luk; } \\
\text { Laura Anderson; } \\
\text { Linda Czypinski; } \\
\text { Courtney Coles; } \\
\text { Catherine M. Walsh. } \\
2017 \\
\text { EUA } \\
\text { Scopus } \\
\text { NE: } 2\end{array}$ & Vídeo & $\begin{array}{l}\text { Avaliação da } \\
\text { mobilidade } \\
\text { física; } \\
\text { Análise de } \\
\text { fármacos. }\end{array}$ & $\begin{array}{l}\text { Redução das quedas; } \\
\text { Construção da visão } \\
\text { crítica sobre o risco } \\
\text { de quedas. }\end{array}$ \\
\hline
\end{tabular}




\begin{tabular}{|c|c|c|c|c|}
\hline $\begin{array}{l}\text { Crew Resource } \\
\text { Management } \\
\text { for Obstetric } \\
\text { and Neonatal } \\
\text { Teams to } \\
\text { Improve } \\
\text { Communicatio } \\
n \text { During } \\
\text { Cesarean } \\
\text { Births }^{23}\end{array}$ & $\begin{array}{l}\text { Mary P. Mancuso; } \\
\text { Oliwier Dziadkowiec; } \\
\text { Catherine Kleiner; } \\
\text { Katherine Halverson- } \\
\text { Carpenter; } \\
\text { Terri Link; } \\
\text { James Barry } \\
2016 \\
\text { EUA } \\
\text { Scopus } \\
\text { NE: } 2\end{array}$ & Vídeo & $\begin{array}{l}\text { Princípios } \\
\text { sobre } \\
\text { treinamento da } \\
\text { equipe estilo } \\
\text { aviação e } \\
\text { gestão de } \\
\text { recursos da } \\
\text { tripulação; } \\
\text { Comunicação } \\
\text { crítica da } \\
\text { linguagem e } \\
\text { trabalho em } \\
\text { equipe. }\end{array}$ & $\begin{array}{l}\text { Melhora na } \\
\text { comunicação entre } \\
\text { profissionais de } \\
\text { saúde entre os } \\
\text { membros da equipe } \\
\text { capacitada. }\end{array}$ \\
\hline $\begin{array}{l}\text { Oral Care } \\
\text { Education in } \\
\text { the Prevention } \\
\text { of Ventilator- } \\
\text { Associated } \\
\text { Pneumonia: } \\
\text { Quality Patient } \\
\text { Outcomes in } \\
\text { the Intensive } \\
\text { Care Unit }{ }^{21}\end{array}$ & $\begin{array}{l}\text { Joyce Zurmehly } \\
2013 \\
\text { EUA } \\
\text { Scopus } \\
\text { NE: } 2\end{array}$ & $\begin{array}{l}\text { Módulo de } \\
\text { educação } \\
\text { online }\end{array}$ & $\begin{array}{l}\text { Melhores } \\
\text { práticas sobre } \\
\text { a pneumonia } \\
\text { associada ao } \\
\text { ventilador } \\
\text { (PAV): } \\
\text { epidemiologia } \\
\text { e escopo do } \\
\text { problema, } \\
\text { fatores de } \\
\text { risco, } \\
\text { definições, } \\
\text { estratégias } \\
\text { para reduzir a } \\
\text { PAV e } \\
\text { prevenção. }\end{array}$ & $\begin{array}{l}\text { Higienização bucal } \\
\text { adequada; } \\
\text { Maior conhecimento } \\
\text { sobre PAV; } \\
\text { Redução dos índices } \\
\text { de PAV na } \\
\text { instituição em } \\
\text { estudo. }\end{array}$ \\
\hline $\begin{array}{l}\text { A cluster } \\
\text { randomised } \\
\text { controlled } \\
\text { feasibility } \\
\text { study of nurse- } \\
\text { initiated } \\
\text { behavioural } \\
\text { strategies to } \\
\text { manage } \\
\text { interruptions } \\
\text { during } \\
\text { medication } \\
\text { administration }{ }^{1} \\
7\end{array}$ & $\begin{array}{l}\text { Maree Johnson; } \\
\text { Rachel Langdon; } \\
\text { Tracy Levett-Jones; } \\
\text { Gabrielle Weidemann; } \\
\text { Elizabeth Manias, } \\
\text { Bronwyn Everett } \\
2019 \\
\text { Escócia } \\
\text { Medline/Pubmed } \\
\text { NE: } 2\end{array}$ & $\begin{array}{l}\text { Módulo de } \\
\text { educação } \\
\text { online }\end{array}$ & $\begin{array}{l}\text { Estratégias } \\
\text { para gerenciar } \\
\text { interrupções } \\
\text { durante a } \\
\text { administração } \\
\text { de } \\
\text { medicamentos; } \\
\text { Comunicação } \\
\text { entre equipe. }\end{array}$ & $\begin{array}{l}\text { Não houve } \\
\text { resultados } \\
\text { significativos; } \\
\text { alguns indicadores } \\
\text { permaneceram com } \\
\text { o mesmo índice na } \\
\text { instituição em } \\
\text { estudo. }\end{array}$ \\
\hline $\begin{array}{l}\text { A qualitative } \\
\text { study of nurses' } \\
\text { perceptions of } \\
\text { a behavioural } \\
\text { strategies } \\
\text { elearning } \\
\text { program to } \\
\text { reduce } \\
\text { interruptions } \\
\text { during } \\
\text { medication }\end{array}$ & $\begin{array}{l}\text { Maree Johnson; } \\
\text { Tracy Levett-Jones; } \\
\text { Langdon; } \\
\text { Gabrielle Weidemann } \\
\text { Elizabeth Manias; } \\
\text { Bronwyn Everett } \\
2018 \\
\text { Austrália } \\
\text { Scopus } \\
\text { NE: } 5\end{array}$ & $\begin{array}{l}\text { Módulo de } \\
\text { educação } \\
\text { online; } \\
\text { Vídeos }\end{array}$ & $\begin{array}{l}\text { Fatores que } \\
\text { incidem sobre } \\
\text { erros e } \\
\text { interrupções } \\
\text { de medicação. }\end{array}$ & $\begin{array}{l}\text { Adesão às } \\
\text { estratégias } \\
\text { comportamentais } \\
\text { para reduzir as } \\
\text { interrupções durante } \\
\text { a administração de } \\
\text { medicamentos. }\end{array}$ \\
\hline
\end{tabular}




\begin{tabular}{|c|c|c|c|c|}
\hline${ }_{4}^{\text {administration }}{ }^{2}$ & & & & \\
\hline $\begin{array}{l}\text { Effectiveness } \\
\text { of an internet- } \\
\text { based learning } \\
\text { program on } \\
\text { venous leg } \\
\text { ulcer nursing } \\
\text { care in home } \\
\text { health care- } \\
\text { study } \\
\text { protocol }^{25}\end{array}$ & $\begin{array}{l}\text { Minna Ylonen; } \\
\text { Jaakko Viljamaa; } \\
\text { Hannu Isoaho; } \\
\text { Kristiina Junttila; } \\
\text { Helena Leino-Kilpi; } \\
\text { Riitta Suhonen } \\
2015 \\
\text { Finlandia } \\
\text { Scopus } \\
\text { NE: } 4 \\
\end{array}$ & $\begin{array}{l}\text { Módulo de } \\
\text { educação } \\
\text { online }\end{array}$ & $\begin{array}{l}\text { Avaliação de } \\
\text { infecção; } \\
\text { Princípios de } \\
\text { tratamento; } \\
\text { Produtos de } \\
\text { tratamento } \\
\text { tópico. }\end{array}$ & $\begin{array}{l}\text { Maior conhecimento } \\
\text { prático; } \\
\text { Uso racional de } \\
\text { suprimentos; } \\
\text { Otimização de } \\
\text { tempo no tratamento. }\end{array}$ \\
\hline $\begin{array}{l}\text { The Effect of } \\
\text { an Online } \\
\text { Continuing } \\
\text { Education } \\
\text { Module on } \\
\text { Nurses' Use of } \\
\text { the Lexi-Comp } \\
\text { Feature of the } \\
\text { Pyxis } \\
\text { MedStation } \\
2000^{26}\end{array}$ & $\begin{array}{l}\text { Maureen Straight } \\
2008 \\
\text { EUA } \\
\text { Scopus } \\
\text { NE: } 4\end{array}$ & $\begin{array}{l}\text { Módulo de } \\
\text { educação } \\
\text { online que } \\
\text { adotou o } \\
\text { Sistema Lexi- } \\
\text { Comp/Pyxis } \\
\text { MedStation } \\
\text { versão 2000 }\end{array}$ & $\begin{array}{l}\text { Melhores } \\
\text { práticas de } \\
\text { enfermagem } \\
\text { na fase de } \\
\text { administração } \\
\text { do processo de } \\
\text { entrega de } \\
\text { medicamentos. }\end{array}$ & $\begin{array}{l}\text { Diminuição dos } \\
\text { erros de } \\
\text { administração de } \\
\text { medicamentos. }\end{array}$ \\
\hline $\begin{array}{l}\text { The Impact of } \\
\text { Educational } \\
\text { Interventions } \\
\text { on Enhancing } \\
\text { Perceptions of } \\
\text { Patient Safety } \\
\text { Culture Among } \\
\text { Jordanian } \\
\text { Senior } \\
\text { Nurses }^{18}\end{array}$ & $\begin{array}{l}\text { Raeda Fawzi } \\
\text { AbuAlRub; } \\
\text { Eyad Hani Abu Alhijaa } \\
2014 \\
\text { EUA } \\
\text { Medline/Pubmed } \\
\text { NE: } 2\end{array}$ & $\begin{array}{l}\text { Módulo de } \\
\text { educação } \\
\text { online }\end{array}$ & $\begin{array}{l}\text { Introdução à } \\
\text { segurança do } \\
\text { paciente; } \\
\text { Fundamentos } \\
\text { de segurança } \\
\text { do paciente; } \\
\text { Trabalho em } \\
\text { equipe e } \\
\text { comunicação; } \\
\text { Introdução à } \\
\text { cultura de } \\
\text { segurança. }\end{array}$ & $\begin{array}{l}\text { Diminuição na taxa } \\
\text { de eventos adversos } \\
\text { na instituição em } \\
\text { estudo; } \\
\text { Trabalho em equipe } \\
\text { adequado; } \\
\text { Melhoria da } \\
\text { comunicação. }\end{array}$ \\
\hline $\begin{array}{l}\text { Using Virtual } \\
\text { Human } \\
\text { Technology in } \\
\text { Perioperative } \\
\text { Team Training } \\
\text { Simulations }^{19}\end{array}$ & $\begin{array}{l}\text { Michele Brunges; } \\
\text { Theresa E. Hughes } \\
2020 \\
\text { EUA } \\
\text { Cinahal } \\
\text { NE: } 2\end{array}$ & $\begin{array}{l}\text { Software; } \\
\text { Módulo de } \\
\text { educação } \\
\text { online }\end{array}$ & $\begin{array}{l}\text { Comunicação } \\
\text { multidisciplina } \\
\text { r; } \\
\text { Melhores } \\
\text { práticas } \\
\text { referente a } \\
\text { hemocompone } \\
\text { ntes e } \\
\text { contagem } \\
\text { correta de } \\
\text { peças } \\
\text { cirúrgicas no } \\
\text { perioperatório. }\end{array}$ & $\begin{array}{l}\text { Comunicação } \\
\text { multidisciplinar } \\
\text { otimizada; } \\
\text { Diminuição dos } \\
\text { eventos adversos } \\
\text { com } \\
\text { hemocomponentes e } \\
\text { contagem correta de } \\
\text { peças no } \\
\text { perioperatório. }\end{array}$ \\
\hline
\end{tabular}

Fonte: Os autores 


\section{DISCUSSÃO}

Os estudos evidenciaram que as tecnologias utilizadas para capacitação dos profissionais de enfermagem foram, softwares, vídeos instrucionais, slides e módulos de educação online, demonstrando o avanço da tecnologia na educação dos profissionais nos serviços de saúde. Estes recursos permitem a disseminação das informações de maneira instantânea, globalizada, e holística para o aprendizado.

O investimento na formação de enfermeiros, técnicos e auxiliares é um dos aspectos cruciais para fortalecimento da cultura de segurança do paciente. Neste sentido, a Educação Permanente em Saúde (EPS) associado às tecnologias, tem sido adotada pelas instituições para desenvolvimento de competências e habilidades. ${ }^{27}$ Portanto, a EPS surge como estratégia para aperfeiçoamento da prática no ambiente de trabalho, proporcionando consolidação do conhecimento. ${ }^{28}$

As tecnologias digitais têm sido empregadas em várias experiências pedagógicas manifestando a relevância da sua aplicabilidade no processo de ensino e aprendizagem, pois associam diversos elementos, como texto, imagens, som e animações em um único recurso para promoção do conhecimento. ${ }^{29}$ Desse modo, são classificadas em metodologias ativas, pois promovem interação, engajamento e autonomia dos profissionais. ${ }^{30}$
Para a consolidação das boas práticas de segurança do paciente, as técnicas de simulação com softwares mostram-se eficazes no processo de aprendizagem, pois podem reproduzir ambientes críticos realísticos, controlado por um instrutor para aprimoramento de técnicas sem causar eventuais danos. Vale ressaltar, que os ambientes se mostram seguros, o que viabiliza repetir a capacitação quantas vezes for necessário. ${ }^{31}$ Dessa maneira, a simulação vem conquistando espaço com maior versatilidade há cerca de quatro décadas, com exemplares operados inicialmente pela indústria (aeronáutica e militar). ${ }^{32}$

Os estudos que abordaram a simulação utilizando software s6,19,26 $^{10 m o}$ intervenção, viabilizaram o aprimoramento de habilidades técnicas e não técnicas, conduzindo para construção do pensamento crítico-reflexivo, compreensão da problemática por meio de fundamentos teórico-científico e análise da prática, estimulando a participação em ações internas para melhoria do processo de trabalho em conformidade com os objetivos organizacionais. ${ }^{33}$

Outras tecnologias digitais utilizadas nos estudos, como vídeos e aulas em ambiente virtual de aprendizagem, também foram destacadas como satisfatórias para capacitação dos profissionais sobre segurança do paciente, pois facilita a transmissão do conhecimento por meio de práticas, atividades 
e situações-problema, quando comparado ao método tradicional. ${ }^{33-34}$ Ainda, os autores desvelam benefícios significativos quanto à utilização destas tecnologias, pois podem ser disponibilizados para acesso a qualquer tempo e localidade, respeitando a individualidade, necessidade e limitações de aprendizagem. ${ }^{30}$

Portanto, diante da diversidade de recursos tecnológicos digitais disponíveis, torna-se imprescindível a seleção adequada da tecnologia, seguindo rigorosamente preceitos pedagógicos e científicos para melhores resultados e fortalecimento da cultura de segurança do paciente.

Aponta-se como limitação desta revisão a inclusão apenas de estudos primários, nos idiomas, inglês, português e espanhol, disponíveis na íntegra.

\section{CONCLUSÕES}

Identificou-se nesta revisão integrativa, que as tecnologias digitais utilizadas para capacitação dos profissionais de enfermagem sobre segurança do paciente, como software, arquivo PPT, vídeo e módulo de educação online, proporcionam aprendizado por meio de múltiplas vertentes dos recursos disponíveis. As tecnologias digitais devem ser amplamente utilizadas para despertar a interatividade e participação, sucedendo aprendizados consolidados para melhores práticas em saúde, bem como, fortalecimento da cultura de segurança do paciente nas organizações.

\section{REFERÊNCIAS}

1 Cestari VRF, Ferreira MA, Garces TS, Moreira TMM, Pessoa VLM de P, Barbosa IV. Aplicabilidade de inovações e tecnologias assistenciais para a segurança do paciente: revisão integrativa TT - Applicability of assistive innovations and technologies for patient safety: integrative review TT - Aplicabilidad de inovaciones y tecnología. Cogitare enferm [Internet]. 2017;22:1-9. Available from: http://fiadmin.bvsalud.org/document/view/wwtq4\%0Ahtt p://fi-admin.bvsalud.org/document/view/jjxsk.

2 Ünver S, Yeniğün SC. Patient Safety Attitude of Nurses Working in Surgical Units: A CrossSectional Study in Turkey. J Perianesthesia Nurs. 2020. Doi: 10.1016/j.jopan.2020.03.012.

3 Hendee WR. To Err is Human: Building a Safer Health System. J Vasc Interv Radiol [Internet]. 2001;12:P112-3. Doi: $\quad 10.1016 /$ S10510443(01)70072-3.

4 Vilela RPB, Castilho V, Jericó M de C, Faria JIL. Educação permanente: tecnologia para a prevenção do erro de medicação TT - Permanent education: technology for the prevention of medication error. Cuid Enferm [Internet]. 2017;11:203-8. Available from: http://www.webfipa.net/facfipa/ner/sumarios/cuid arte/2017v2/203.pdf.

5 Lavich CRP, Terra MG, Arnemann CT, Mello AL, Raddatz M. Health education and permanent education: Actions integrating the educational process of nursing. Rev Baiana Enferm. 2018;32:1-10. Doi: 10.18471/rbe.v32.24719.

6 En E, Ciudadanía SY, Integradora R. Educação em saúde e cidadania revisão integrativa. 2014;363-78. Doi: $\quad$ 10.1590/S198177462014000200009 .

7 Mallmann DG, Galindo Neto NM, De Carvalho Sousa J, Vasconcelos EMR. Health education as the main alternative to promote the health of the elderly. Cienc e Saude Coletiva. 2015;20:176372. Doi: 10.1590/1413-81232015206.02382014.

8 Bussotti EA, Leite MTM, Alves AC da C, Cristensen K. Capacitação on-line para profissionais da saúde em três regiões do Brasil. Rev Bras Enferm. 2016;69:981-5. Doi: 10.1590/0034-7167.2016690506. 
9 Girão ALA, Sampaio RL, Aires SF, Oliveira ICL de, Oliveira SKP de, Carvalho REFL de. Medsafe: Prototype of a Virtual Game on the Preparation and Administration of Medications. Reme Rev Min Enferm. 2019;23:1-8. Doi: 10.5935/1415-2762.20190087.

10 Domingues AN, Jesus ITM de, ZemMascarenhas SH. Informática na Educação em Saúde e Enfermagem: análise dos grupos de pesquisa. J Heal Inf. 2017;9:[19-24].

11 Dunwoody CJ, Krenzischek DA, Pasero C, Rathmell JP, Polomano RC. Assessment, Physiological Monitoring, and Consequences of Inadequately Treated Acute Pain. Pain Manag Nurs. 2008;9:11-21. Doi: 10.1016/j.jopan.2007.11.007.

12 Mendes KDS, Silveira RC de CP, Galvão CM. Revisão integrativa: método de pesquisa para a incorporação de evidências na saúde e na enfermagem. Texto Context - Enferm. 2008;17:758-64. Doi: 10.1590/S010407072008000400018 .

13 Santos CM da C, Pimenta CA de M, Nobre MRC. The PICO strategy for the research question construction and evidence search. Rev Lat Am Enfermagem. 2007;15:508-11. Doi: 10.1590/S0104-11692007000300023.

14 Fabbri S, Hernandes E, Di Thommazo A, Belgamo A, Zamboni A, Silva C. Managing Literature reviews information through visualization. ICEIS 2012 - Proc 14th Int Conf Enterp Inf Syst. 2012;2 ISAS:36-45. Doi: $10.5220 / 0004004000360045$.

15 Melnyk BM, Fineout-Overholt E. EvidenceBased Practice in Nursing and Healthcare: A Guide to Best Practice. Philadelphia: Lippincott Williams \& Wilkins, 2011. Chapter 1: Making the case evidence-based practice and cultivating a spirit of inquiry, p. 3-24.

16 Glaser B, Schellenberg T, Koch L, Hofer M, Modemann S, Dubach P, et al. Design and evaluation of an interactive training system for scrub nurses. Int J Comput Assist Radiol Surg. 2016;11:1527-36. Doi: 10.1007/s11548-0161356-9.

17 Johnson $\mathrm{M}$, Langdon $\mathrm{R}$, Levett-Jones $\mathrm{T}$, Weidemann G, Manias E, Everett B. A cluster https://doi.org/10.31011/reaid-2021-v.95-n.34-art.1105 Rev Enferm Atual In Derme v. 95, n. 34, 2021 e-021090 randomised controlled feasibility study of nurseinitiated behavioural strategies to manage interruptions during medication administration. Int J Qual Heal care J Int Soc Qual Heal Care. 2019;31:G67-73. Doi: 10.1093/intqhe /mzz007.

18 Abualrub RF, Abu Alhijaa EH. The impact of educational interventions on enhancing perceptions of patient safety culture among Jordanian senior nurses. Nurs Forum. 2014;49:139-50. Doi: 10.1111/nuf.12067.

19 Brunges M, Hughes TE. Using Virtual Human Technology in Perioperative Team Training Simulations. AORN J. 2020;111:617-26. Doi: 10.1002/aorn.13046.

$20 \mathrm{Lu}$ MC, Yu S, Chen IJ, Wang KWK, Wu HF, Tang FI. Nurses' knowledge of high-alert medications: A randomized controlled trial. Nurse Educ Today [Internet]. 2013;33:24-30. Doi: 10.1016/j.nedt.2011.11.018.

21 Zurmehly J. Oral care education in the prevention of ventilator-associated pneumonia: Quality patient outcomes in the intensive care unit. J Contin Educ Nurs. 2013;44:67-75. Doi: 10.3928/00220124-20121203-16.

22 Nuckols TK, Needleman J, Grogan TR, Liang LJ, Worobel-Luk P, Anderson L, et al. Clinical Effectiveness and Cost of a Hospital-Based Fall Prevention Intervention: The Importance of Time Nurses Spend on the Front Line of Implementation. J Nurs Adm. 2017;47:571-80. Doi: $10.1097 /$ NNA.0000000000000545.

23 Mancuso MP, Dziadkowiec O, Kleiner C, Halverson-Carpenter K, Link T, Barry J. Crew Resource Management for Obstetric and Neonatal Teams to Improve Communication During Cesarean Births. JOGNN - J Obstet Gynecol Neonatal Nurs [Internet]. 2016;45:502-14. Available from: http://dx.doi.org/10.1016/j.jogn.2016.04.006

24 Johnson $\mathrm{M}$, Levett-Jones $\mathrm{T}$, Langdon $\mathrm{R}$, Weidemann G, Manias E, Everett B. A qualitative study of nurses' perceptions of a behavioural strategies e-learning program to reduce interruptions during medication administration. Nurse Educ Today. 2018;69:41-7. Doi: 10.1016/j.nedt.2018.06.028.

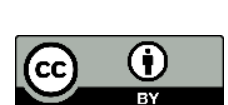


25 Ylönen M, Viljamaa J, Isoaho H, Junttila K, Leino-Kilpi H, Suhonen R. Effectiveness of an internet-based learning program on venous leg ulcer nursing care in home health care - study protocol. J Adv Nurs. 2015;71:2413-25. Doi: 10.1111/jan.12683.

26 Straight M. One strategy to reduce medication errors: The effect of an online continuing education module on nurses' use of the LexiComp feature of the Pyxis MedStation 2000. CIN - Comput Informatics Nurs. 2008;26:23-30. Doi: 10.1097/01.NCN.0000304761.56671.ad.

27 Salum NC, Prado ML. A educação continuada permanente no desenvolvimento de competências aos profissionais de enfermagem. Texto e Context Enferm. 2014;23:301-8. Doi: 10.1590/0104070720140021600011 .

28 Meneses IG, Alves Junior EDD, Santos ABG dos, Pereira AV, Domingos AM, Corvino MPF. Educação permanente em equipe multidisciplinar de um programa gerontológico: concepções, desafios e possibilidades. ABCS Heal Sci. 2019;44:40-6. Disponível em: https://docs.bvsalud.org/biblioref/2019/05/995025 /44abcs40.pdf.

29 Dalmolin A, Girardon-Perlini NMO, Coppetti L de C, Rossato GC, Gomes JS, Silva MEN da. Vídeo educativo como recurso para educação em saúde a pessoas com colostomia e familiares. Rev Gauch Enferm. 2017;37:e68373. Doi: 10.1590/1983-1447.2016.esp.68373.

30 Góes F dos SN de, Camargo RAA de, Fonseca LMM, Oliveira GF de, Hara CYN, Felipe HR, et al. Assessment of the Digital Educational Technology "Vital Signs and Anatomy" By Students of Vocational Nursing Education. REME Rev Min Enferm. 2015;19:37-43. Doi: 10.5935/1415-2762.20150024.

31 Miranda RPR, Motta AL, Chaves E de CL, Resck ZMR, Iunes DH. A aplicabilidade do uso de Simulação Realística na Formação Permanente do Profissional de Enfermagem. Rev Interdiscip Estud em Saúde. 2016;4:54-62. Doi: 10.33362/ries.v4i2.713. Doi: 10.33362/ries.v4i2.713.

32 Cooper JB, Taqueti VR. A brief history of the development of mannequin simulators for clinical education and training. Postgrad Med J. https://doi.org/10.31011/reaid-2021-v.95-n.34-art.1105 Rev Enferm Atual In Derme v. 95, n. 34, 2021 e-021090
2008;84:563-70.
Doi:
10.1136 qshc.2004.009886.

33 Campoi ALM, Engel RH, Stacciarini TSG, Cordeiro ALP de C, Melo AF, Rezende MP. Educação permanente para boas práticas na prevenção de lesão por pressão: quaseexperimento. Rev Bras Enferm. 2019;72:1725-31. Doi: 10.1590/0034-7167-2018-0778.

34 Prado C, Vaz DR, Almeida DM de. Teoria da aprendizagem significativa: elaboração e avaliação de aula virtual na plataforma Moodle. Rev Bras Enferm. 2011;64:114-1121. Doi: 10.1590/S0034-71672011000600019.

\section{Autor correspondente}

Nome:Valeska Tais de Araújo Hoffmann Endereço: Rua Espírito Santo, 772 CEP: 86.010-510

Telefone: (43) 9 9855-0516 Email: hoffmannvaleska@gmail.com

Submissão: 2021-05-06

Aprovado: 2021-05-30 